\title{
Biographisches und Kulturhistorisches aus Briefen und Akten von Ludwig Schläfli
}

Von Erwin Neuenschwander

Ludwig Schläfli zählt zu den wenigen bedeutenden Schweizer Mathematikern der vergangenen Jahrhunderte, deren Wirken zeit ihres Lebens mit der Schweiz verbunden blieb. An dieser Stelle sollen neun in kulturhistorischer und biographischer Hinsicht aufschlußreiche Schläfli-Briefe aus dem Nachlaß von Felice Casorati veröffentlicht werden als Ergänzung zu unserer vorangegangenen mathematischen Studie über diesen Nachlaß. Gleichzeitig benützen wir die Gelegenheit, um gewisse in den bisherigen Biographien meist fehlerhaft wiedergegebene Abschnitte aus Schläflis Leben zu klären, und publizieren hierzu vier Dokumente aus den in Berner Archiven liegenden Schläfli-Akten. Zur Einleitung schicken wir einige Angaben über Leben und Werk von Schläfli, eine kurze Übersicht über seine Beziehungen zu Casorati sowie einen ausführlichen Kommentar mit Inhaltsangabe zum gesamten Briefwechsel zwischen den beiden Mathematikern voraus.

\section{Leben und Werk von Ludwig Schläfli}

Ludwig Schläfli wurde am 15. Januar 1814 in Graßwil (Kt. Bern) als ältestes von vier Kindern des Negotianten Johann Ludwig Schläfli-Aebi geboren ${ }^{1}$. Er besuchte zunächst die Schulen in Burgdorf, sodann ab 1829 mit einem Stipendium das Gymnasium in Bern und trat später, dem Wunsche seiner relativ früh verstorbenen Eltern entsprechend, in die theologische Fakultät der Universität Bern ein $^{2}$. Im Wintersemester 1836/37 absolvierte er einen Teil seines theologischen Examens und nahm danach, seiner Veranlagung folgend, eine Lehrstelle für Mathematik und Naturlehre an der Stadtschule in Thun an. Dort wurde er zunächst provisorisch auf ein Jahr angestellt, und zwar für 22 wöchentliche Unterrichtsstunden bei einer jährlichen Besoldung von 700 a. Fr. ${ }^{3}$ nebst Wohnung und Heizung ${ }^{4}$. Während der nächsten zwei Jahre reiste Schläfli einmal wöchentlich nach Bern, um seine theologischen Studien abzuschließen ${ }^{5}$. Nachdem er jedoch im Herbst 1838 sein theologisches Staatsexamen samt der Probepredigt bestanden hatte ${ }^{6}$, trat er nicht ins Pfarramt ein, sondern unterrichtete weiterhin an dem inzwischen aus der Stadtschule hervorgegangenen Thuner Progymnasium. Im Jahre 1839 (nicht 1838) wurde Schläfli an dieser Schule zum «definitiven» 
Lehrer für Mathematik und Naturkunde ernannt ${ }^{7}$ für 21 Wochenstunden mit einer jährlichen Besoldung von 1050 a. Fr. ${ }^{3}$ nebst Wohnung ${ }^{8}$.

Über Schläflis Lehrtätigkeit am Progymnasium Thun sind wir dank der jährlichen Examensberichte und den erhaltenen Protokollen von den Sitzungen des Verwaltungsrates verhältnismäßig gut unterrichtet. In allen Akten werden Schläfli zwar durchaus gründliche Kenntnisse in seinen Unterrichtsfächern zugebilligt, jedoch wird von Anfang an immer wieder betont, daß sein Unterricht «zu hoch gehalten» sei und die Fassungskraft der Schüler übersteige ${ }^{9}$. Gegen Ende von Schläflis Thuner Jahren mehren sich diese Klagen, wobei zusätzlich auch die Disziplinlosigkeit der Schüler in seinen Schulstunden getadelt wird (vgl. hinten, Akten 1 und 2). Es wäre somit möglich, daß diese steten Rügen Schläflis Entschluß bestärkten, sich nach einer seiner Persönlichkeit besser entsprechenden Tätigkeit umzusehen ${ }^{10}$. Im September 1843 ersuchte er den Verwaltungsrat um Gewährung eines «Urlaubs für den Winter 1843-44, zum Behuf des Besuchs der Universität Berlin ». ${ }^{11}$ Sein kongenialer Landsmann Jakob Steiner, der damals in Bern gerade auf der Durchreise weilte, riet ihm jedoch von diesem Plan ab, da in Berlin zur Zeit niemand anzutreffen sei. Steiner lud Schläfli statt dessen ein, zusammen mit ihm, C. W. Borchardt, G. Lejeune Dirichlet und C. G. J. Jacobi als Dolmetscher nach Rom zu reisen ${ }^{12}$.

Der halbjährige Italienaufenthalt war für den Autodidakten Schläfli von entscheidender Bedeutung, da er ihm endlich ermöglichte, mit den ersten Männern seines Faches unmittelbar in Kontakt zu treten. Im Frühjahr 1847 (nicht 1848) verließ Schläfli seine Thuner Lehrstelle und wurde Privatdozent an der Universität Bern, wo er 1853 zum Extraordinarius und 1872 (nicht 1868 oder 1871) ${ }^{13}$ zum Ordinarius befördert wurde (vgl. hinten, Akten 3 und 4) ${ }^{14}$. An der Berner Universität mußte Schläfli während langer Zeit «im eigentlichen Sinne des Wortes darben ». ${ }^{15}$ Zunächst erhielt er nur das gesetzliche Dozentengehalt von 400 a. Fr. ${ }^{3}$ pro Jahr und erreichte selbst im Alter von 48 Jahren, der geringen Hörerzahlen wegen, erst 1400 Fr., was der Besoldung eines unteren Kanzlisten entsprach $^{16}$. Schläfli konnte sich damals nur über Wasser halten durch Privatlehrertätigkeit und als Liquidationsrechner bei der Massaverwaltung der schweizerischen Nationalvorsichtskasse, ein Nebenerwerb, den ihm sein Freund Friedrich Ris $^{17}$ vermittelt hatte ${ }^{18}$. Unter dem Druck von Schläflis Schülern wurde seine Besoldung schließlich auf 4000 Fr. pro Jahr angehoben, womit er den übrigen Professoren einigermaßen gleichgestellt war (vgl. hinten, Akte 4). Nach 45 Jahren Lehrtätigkeit an der Universität Bern trat Schläfli Ende 1891 wegen seines sich ständig verschlechternden Gesundheitszustandes zurück und starb einige Jahre später am 20. März 1895 in Bern. 
Ludwig Schläfli war ein äußerst vielseitiger Mathematiker, der nie bloß bestimmte Sachgebiete bearbeitete, sondern stets mathematische Ideen entwickelte, deren Ausarbeitung in verschiedene Disziplinen hineinreichte. Seine Hauptverdienste liegen auf den Gebieten der Theorie der Elimination, der höheren Geometrie und der Funktionentheorie. Schläflis gedruckte Abhandlungen wurden beinahe vollständig in den Jahren 1950-1956 mit Kommentar in einer dreibändigen Werkausgabe von der Schweizerischen Naturforschenden Gesellschaft ediert $^{19}$. Die auch heute noch umfassendste Schläfli-Biographie stammt vom bernischen Mathematiker und Wissenschaftshistoriker Johann Heinrich Graf, der Schläflis Schüler und Nachfolger war und ihn auch persönlich ausgezeichnet kannte $^{20}$. In Grafs Biographie findet man eine ausführliche Lebensbeschreibung (S.120-147), eine Übersicht über Schläflis Korrespondenz (S.147-153), eine Zusammenstellung der Nekrologe (S.154-156), eine Liste seiner Abhandlungen und nachgelassenen Manuskripte (S.157-178) sowie ein Verzeichnis der von Schläfli an der Berner Universität angekündigten Vorlesungen (S.179-203). Ferner hat Graf auch Schläflis Briefwechsel mit Steiner, Cayley, Borchardt und den italienischen Mathematikern (Chelini, Beltrami, Casorati und Cremona) ediert, wobei er sich jedoch fast immer auf die in Bern archivierten Briefe beschränkte. In neuerer Zeit hat sich vor allem Johann Jakob Burckhardt um Schläfli verdient gemacht, indem er seinen mathematischen Nachlaß bearbeitete und bei der Edition von Schläflis Werken mithalf. In Burckhardts Arbeiten findet man eine eingehende mathematische Würdigung von Schläflis Publikationen, die zum Teil durch Albert Häusermann ergänzt wurde.

\section{Schläflis Freundschaft und Briefwechsel mit Felice Casorati}

Mit Felice Casorati (1835-1890) ${ }^{21}$ verband Schläfli eine tiefe Freundschaft, wovon der ausgedehnte Briefwechsel (über 100 Briefe von 1869 bis 1890) und die vielen gemeinsam verbrachten Ferienaufenthalte der beiden Mathematiker ein beredtes Zeugnis ablegen. In den Jahren 1869, 1871-1877 besuchte Schläfli jeweils im Spätsommer während mehrerer Wochen Casorati, der an den oberitalienischen Seen mit seiner Familie in einer Ferienwohnung weilte ${ }^{22}$, um der drückenden Hitze an seinem Wohnort Pavia zu entgehen. Als Schläfli später einen eigenen Haushalt begonnen hatte und wegen der anfänglichen Krankheiten seiner Haushälterin Bern nicht mehr ohne weiteres für längere Zeit verlassen wollte, trafen sich die beiden Freunde noch zweimal in den Schweizer Alpen (in Parpan im August 1879 und in Zuoz im August 1882). Schläfli pflegte im Anschluß an diese Begegnungen Casorati jeweils seine gute Rückkehr nach Bern 
anzuzeigen, wobei er zum Teil kulturhistorisch aufschlußreiche Reiseschilderungen beifügte. Weiter findet man in Schläflis Briefen Angaben über seine Haushaltsgründung, seine Haushälterin, seine Freunde, seine Vorlesungen sowie seine Altersgebresten. Schläflis Briefwechsel mit Casorati ist in biographischer Hinsicht auch darum von Bedeutung, weil die bisher publizierten Schläfli-Briefe vor allem aus der vorangehenden Periode 1853-1877 stammen.

Casoratis Briefe an Schläfli liegen im Schläfli-Nachlaß der Schweizerischen Landesbibliothek in Bern und wurden durch Graf 1916 und 1917 (Briefe Nr.1-2 und 4-51) publiziert. Dabei sind Graf allerdings einige Briefe entgangen, wie sich aus den in Pavia liegenden Briefentwürfen von Casorati und den im Briefwechsel enthaltenen Angaben ergibt. Schläflis Briefe befinden sich im Casorati-Nachlaß in Pavia, der zur Zeit von Prof. Alberto Gabba betreut wird. Publiziert sind davon bis jetzt nur zwei Briefentwürfe bei Graf 1916 (Brief Nr. 3) und Graf 1917 (Brief Nr. 52), sieben Auszüge aus mathematisch gehaltvollen Briefen bei Neuenschwander 1978 (Briefe Nr. 27-33) sowie die in dieser Arbeit S. 284-293 I wiedergegebenen Briefe Nr.1-9. Auf noch nicht veröffentlichte Briefe wird im nachfolgenden Kommentar jeweils unter Nennung des Abfassungsdatums verwiesen.

\section{Kommentar zu den ausgewählten Briefen und zum Briefwechsel im allgemeinen}

Brief Nr. 1 vom 15. Oktober 1872 beschreibt den zweiten Teil von Schläflis Rückreise nach Bern nach seinem etwa sechswöchigen Besuch bei Casorati in Gravedona am Comersee. Die Rückreise erfolgte damals über Menaggio, Porlezza, Lugano, Bellinzona, Airolo und Luzern und dauerte wegen der schlechten Verbindungen etwa 6 Tage $^{23}$. Das Reisen in den Postkutschen war oft so beschwerlich, daß man davon sogar Zahnschmerzen bekommen konnte, wie Schläfli in einem früheren Brief vom 24. Oktober 1871 schildert. Dort liest man: «...; der Postwagen hatte drei Supplemente, diese sind übel ein gerichtet, bald fehlten die Fenster, bald schlossen die Thüren nicht, zwischen dem Hospiz und Hospenthal gieng dem Beiwagen (Supplement), in dem ich fuhr, ein Rad ab; bei jeder Station werden die Beiwagen gewechselt, die Passagiere müssen sich selbst in den neuen Beiwagen ihre Plätze suchen, denn der Conducteur ist schon ab gefahren. Ich bekam wirklich von der Heimreise für einige Tage Zahnweh mit Geschwulst; ...». Schläfli zog es daher häufig vor, zu Fuß zu gehen, da er so die Natur und die Pflanzen besser beobachten konnte (vgl. hierzu Brief Nr. 6, Abschnitt 1; Graf 1895, S. 141, sowie Rytz 1919, S. 214, der Schläflis botanische Exkursionen und seine Verdienste um die Berner Flora eingehend gewürdigt hat). Von Schläflis naturwissenschaftlichen Neigungen zeugen auch seine Ausführungen im Brief 
Nr. 8 über Oswald Heers ${ }^{24}$ Buch «Die Urwelt der Schweiz», seine eingehende Erörterung des kürzesten Reiseweges von Mailand nach Interlaken (Brief vom 3. Juli 1877) sowie seine wiederholte Bitte an Casorati, ihm die geographische Lage seines Ferienaufenthaltsortes, der Valle di Scalve, zu schildern (Graf 1916, Brief Nr. 37). Von einer längeren Fußreise von Samaden über den Albulapaß, Thusis, Reichenau, Ilanz, Oberalppaß und Susten nach Meiringen berichtet Schläfli in einem Brief vom 1.September 1869 und stellt dabei fest: «... auf meinem ganzen Wege von Samaden bis zum Ursernthal war alles romanisch mit Ausnahme von Thusis und eines vereinzelten Dorfes (Obersaxen oder Surseiz) am Abhange des Piz Mundaun, ...».

Brief Nr. 2 vom 10. August 1876 berichtet uns über die Haushaltungsgründung Schläflis. Graf 1895, S.135, schreibt in diesem Zusammenhang: «Er wohnte die längste Zeit im Haus Gränicher (ehemals Café Dumont) neben der Oranienburg, einmal auch im Sprengergut in Wabern und als das Café Gräf, in dem er zu speisen gewohnt war, geschlossen wurde, hatten wir das Glück, ihn zirka ein Jahr lang an unserm Mittagstische zu sehen. Ein bürgerlicher Haushalt hat ihm daraufhin so gut gefallen, dass er sich entschloss eine eigene Haushaltung anzufangen und dabei hatte er das Glück, in Fräulein Margar[etha] Spichtin eine Haushälterin zu finden, die für ihn, da er so gar nichts auf die äussere Erscheinung gab, in ausgezeichneter Weise besorgt war.» Aber auch Schläfli sorgte in geradezu rührender Weise für seine Haushälterin, die anfänglich häufig krank war (vgl. Briefe Nr. 3-5 ${ }^{25}$ ). Der anfänglich schlechte Gesundheitszustand der Haushälterin war auch eine der Ursachen, weshalb Schläfli Casorati von 1877 an nicht mehr in Italien besuchen wollte. Am 24. Juli 1879 begründete er seine Absage mit folgenden Worten: «Fern von Bern, wäre ich wegen meiner Haushälterin in beständiger Todesangst» (Brief Nr. 5, Abschnitt 2). Schläfli hat seiner Haushälterin, die ihn bis zu seinem Tode treu pflegte (vgl. hierzu Brief Nr.9), übrigens sein bescheidenes Vermögen vermacht (Graf 1895, S. 146).

Brief Nr. 5 vom 24. Juli 1879 erzählt uns von Schläflis Mißgeschick bei einer Wohnungsänderung. In zwei nachfolgenden Briefen schildert Schläfli die von seiten des Hausherrn und seiner Familie ihm zugefügten Feindseligkeiten genauer. Am 16. August 1879 schreibt er: «Nun stehen die Hausfrau und ihre Kinder zu mir und meiner Haushälterin in einem feindseligen Verhältniss. Wir haben uns vor genommen, dass immer eines von uns die Wohnung hüte.» Am 4. November 1879 vermerkt er: «Während meiner Abwesenheit machte meine Haushälterin eine Wasche; die Hausherrin, die meine Abreise bemerkt hatte, sperrte ihr während eines halben Tages aus Bosheit das Wasser ab.» Schläfli scheint auch in späteren Jahren bei der Wohnungssuche nicht immer Glück ge- 
habt zu haben. Von 1876 bis 1888 wechselte er seine Wohnung mindestens siebenmal (vgl. dazu die verschiedenen Adressen am Ende der hier publizierten Briefe).

Schläfli war zwar ein herzensguter, jedoch höchst unpraktischer und verschlossener Mensch. Nur wenigen gelang es, in seine «Weltvergessenheit» einzudringen ${ }^{26}$. Als Casorati ihn am 29. Dezember 1887 (Graf 1916, Brief Nr. 46) ermunterte, doch etwas vermehrt gesellschaftlichen Umgang zu pflegen, entgegnete ihm Schläfli: «Ein Mal habe ich keine gesellschaftliche Anlage, und zum andern sind meine meisten Bekannten schon todt.» (Brief Nr. 7 vom 7.Januar 1888.) Schläfli dachte dabei wohl vor allem an seinen Freund Friedrich Ris ${ }^{17}$, von dessen Tod wir im gleichen Brief erfahren. Schläfli wurde schon als junger Student seit dem Tode seiner Eltern durch Ris unterstützt, der «überhaupt immer wie ein zweiter Vater» für ihn sorgte (vgl. Brief Nr. 6, Abschnitt 3, sowie Graf 1895, S. 124, 130 f.; Graf 1896, S. 61 f., 102 f., und Feller 1935, S. 273).

Bei festlichen Anlässen fühlte sich Schläfli im allgemeinen nicht wohl. Zwar hatte er das Reale Istituto Lombardo di Scienze e Lettere von Mailand anläßlich der Feier zum hundertjährigen Todestag Albrecht von Hallers am 12. Dezember 1877 in Bern vertreten (vgl. seinen Bericht im Brief Nr. 4, Abschnitt 2); als ihn aber Casorati auch zur Hochzeit seiner Tochter Eugenia einladen wollte, entgegnete ihm Schläfli: «Ich bin im gesellschaftlichen Leben zu unerfahren, um den Glanz eines Festes durch meine Anwesenheit erhöhen zu können.» (Brief vom 20.September 1883.) Die soeben zitierte Briefstelle beweist auch Schläflis große Bescheidenheit. Er verstand es höchst selten, Erfolge äußerlich auszunützen, und stellte meist die Leistungen anderer über seine eigenen (vgl. z. B. Brief Nr. 9, Abschnitt 3, oder auch Graf 1895, S. 120, 130 usw.). Als ihm Casorati (Graf 1916, Brief Nr. 40) die Ernennung zum korrespondierenden Mitglied der Reale Accademia dei Lincei in Rom anzeigte, bedankte sich Schläfli mit den Worten: «Sie senden mir eine ganz unerwartete Kunde. Weder durch meine bisherigen Leistungen habe ich eine solche Auszeichnung verdient, noch werde ich sie durch künftige Leistungen verdienen können.» (Brief vom 5. Dezember 1883.)

Auf seine Vorlesungen bereitete sich Schläfli stets sorgfältig vor, obwohl er nur wenige Zuhörer hatte, wie verschiedene Stellen in seinem Briefwechsel zeigen $^{27}$. Am 7. August 1880 schreibt er zum Beispiel an Casorati: «Diesen Sommer habe ich mit meinen Vorlesungen viele Arbeit und wenige Zuhörer gehabt; ich habe sie bis gegen Ende Juli fort gesetzt und bin wohl der letzte Docent gewesen, der aus dem Hochschulgebäude heraus gieng." Schläflis Zuhörer waren jedoch meist Gymnasiallehrer, Privatdozenten und Professoren (Feller 1935, S. 223) und nötigten ihn immer wieder, über Gebiete vorzutragen, die ihm selbst noch 
unbekannt waren (Brief vom 17. September 1881), was die große Vorbereitungszeit erklärt. Schläflis Einsatz wurde jedoch viele Jahre nicht belohnt, da der damalige Erziehungsdirektor Kummer die Professorengehälter anhand des Produktes aus gelesenen Stunden mal Hörerzahl festlegte.

Die Briefe Nr. 7-9 aus den Jahren 1888 und 1889 schildern in offener Weise die allmähliche Abnahme von Schläflis körperlichen und geistigen Kräften. Am 7. Januar 1888 bemerkt Schläfli anläßlich des Todes seines Freundes Ris: «Ich habe nun den Verlauf des Alters deutlich vor Augen ...». Am 9. Juli beklagt er sich über sein rheumatisches Übel, gegen welches ihm der Arzt zunächst Sodabäder verschrieb. Im Januar 1889 war sein Gesundheitszustand so arg geworden, daß er seine Vorlesungen mitten im Semester abbrechen mußte (vgl. Neuenschwander 1978, Brief Nr. 33) und während dreier Wochen nach Baden zur Kur ging (Brief vom 3. August 1889).

Die Abnahme von Schläflis geistigen und körperlichen Kräften klingt im Briefwechsel erstmals in einem Schreiben vom 17. Mai 1874 an, wo Schläfli Casorati zu bedenken gibt: «... ich habe das 60 ste Jahr überschritten, mein Schädel ist verhärtet und für neue Begriffe schwer zugänglich » (Neuenschwander 1978, Brief Nr. 29). Im Jahre 1878 beklagt er sich über «widerholte Anfälle von Unwohlsein» (Brief Nr.4) und im Jahre 1880 über «die Langsamkeit im denken» (Brief vom 26. Dezember 1880), doch scheint sich sein Gesundheitszustand damals wieder etwas gebessert zu haben. Im Februar $1886^{28}$ setzte dann sein Rheumatismus ein, worüber er Casorati am 9. September 1887 berichtet: «... mein Gesundheitszustand ist in diesem Augenblicke wenigstens nicht gar köstlich. Ich halte es für besser, dass ich meine schwachen Kräfte zusammen halte und täglich zur Erfüllung meiner Obligenheiten verwende. Augen, Gehör, Aufmerksamkeit sind schwach, und seit dem Ende febr. 86 habe ich rheumatische Erscheinungen, die mir völlig neu waren und mir sagen, dass ich nicht zu viel wagen solle.» Schläflis Beschwerden wurden zusehends stärker (vgl. oben), so daß er den Regierungsrat im Jahre 1891 um seine Entlassung bat (vgl. hinten, Akte 4) und wenig später am 20. März 1895 starb (vgl. Graf 1895, S. 141 ff. und 202 f.).

\section{Ausgewählte Briefe von L.Schläfli an F. Casorati}

Die einzelnen Briefe sind im allgemeinen manuskriptgetreu wiedergegeben. Schläflis schwankende Verwendung von ss und $\beta$ wurde jedoch stets durch ss wiedergegeben, $\overline{\mathrm{m}}$ durch $\mathrm{mm}$, $\mathrm{u}$ durch $\ddot{\mathrm{u}}$. Unterstrichene Wörter werden durch Kursivdruck gekennzeichnet, Ergänzungen durch eckige Klammern. 
Bern 15 Oct. 1872.

Theurer Freund!

Der Übergang über den Gotthard war merkwürdig. Am $13^{\text {ten }}$ dauerte die Fahrt von $8 \mathrm{Uhr}$ Morgens bis $3 \mathrm{Uhr}$ Nachmittags, von Airolo bis zum Hospiz hinauf. Die Diligence bestund aus drei Hauptwagen und drei Beiwagen. Vor jedem der zwei ersten Hauptwagen waren acht, vor dem dritten sechs Pferde angespannt; jeder der drei Beiwagen hatte drei Pferde. Dem Zug giengen vier Schneeschaufler voran. Die grösste Schwierigkeit machten immer die Wendungen; die drei vordersten Pferde wurden abgespannt; die übrigen brachten mit grosser Mühe den Wagen über die Wendung hinaus; und dann wurden jene drei Pferde wieder angespannt. Der Wagenzug hielt jedes Mal still, bis die Schneeschaufler ihre Arbeit vollendet hatten. Der Conducteur hatte mir in Airolo einen guten Platz im Coupé des zweiten Hauptwagens angewiesen. Einmal entrannen die drei vordersten Pferde dieses Wagens, nachdem sie abgespannt waren, und waren bald aus meinem Gesichtsfeld verschwunden; es dauerte lange, bis man sie wieder zurück gebracht hatte, und ohne sie konnte der Wagen nicht vorwärts. Im Hospiz war eine ausgezeichnete Mittagstafel gedeckt. Von hier gieng der Zug ohne weitere Schwierigkeit ins Reussthal hinunter und langte Abends um 10 Uhr in Flüelen an. Der Schnee bedeckte nur das Urserenthal und hörte unterhalb der Schöllenen auf. Eine Fussreise über den Susten nach Meiringen wäre mir jedenfalls unmöglich gewesen. Ich bekam kein Zahnweh, war aber auch besser gekleidet als voriges Jahr bei meinem Übergang über den Gotthard, und bin ganz gesund in Bern angelangt.

Ich wollte in Kürze Ihnen meine Rückkunft anzeigen, und werde Ihnen bald mehreres schreiben.

Sie und Ihre Gesellschaft freundschaftlich grüssend

Ihr treuer

L. Schläfli

Nr. 2: Brief vom 10. August 1876, 2 Seiten

Theurer Freund!

So manches Jahr habe ich schon Ihre Gastfreundschaft genossen und fröhliche Tage in Ihrer theuren Gesellschaft verlebt; Sie werden sich billig verwundern, dass ich dieses Mal Ihrer freundlichen Einladung nicht entspreche. Wie ich schon an Frau Vistarini ${ }^{29}$ geschrieben habe, bin ich im April in Berlin gewesen während 
3 Wochen, habe dann meine Wohnung verändert und eigenen Haushalt an gefangen; zu meiner neuen Wohnung gehört ein kleines Stück Garten, wo ich Kohl, Sellerie, Kresse, Lauch, ... gepflanzt habe; endlich habe ich wirklich viele Zeit nöthig um mich auf die Wintervorlesungen vor zu bereiten; ich muss die diesen Sommer angefangene Theorie der Integrale algebraischer Functionen fort setzen.

Wenn Sie nächstes Jahr auch wieder einen Aufenthalt am Fusse der Alpen zu machen gedenken und mir Ihr grosses Wohlwollen auch ferner bewahren, so wird es mich sehr freuen, einige Tage in Ihrer werthen Gesellschaft zu verweilen und Luft und Licht auf Italiens Boden zu geniessen.

Haben Sie meinen herzlichen Dank für das werthvolle Geschenk, das Sie mir zugesandt haben.

Grüssen Sie mir die Frau Vistarini und die zwei Töchter Cavalletti ${ }^{29}$, und seien Sie herzlich gegrüsst von

Bern 10 Aug. 1876

Ihrem ergebenen Freunde

Brunnadern $\mathrm{N}^{\circ} 11$ c.

L. Schläfli

\section{Nr. 3: Brief vom 15. November 1876, 1 Seite}

Theurer Freund!

Als ich Ihnen das letzte Mal in aller Eile über meine Ankunft Bericht gab, glaubte ich wirklich, ich werde Ihnen noch vor Ablauf des Oct. nach Rezzonico schreiben. Vor etwa einer Woche hat sich endlich die schwere Krankheit meiner Haushälterin gewendet, das Fieber hörte auf; und sie fieng nun an zu essen; jetzt steht sie wider alle Tage auf, hat aber über Tag noch immer eine Krankenwärterin bei sich, die zugleich die Kocherei und meine Bedienung besorgt. Der Arzt kam täglich ins Haus, bis er selbst krank ward. Er erklärte die Krankheit für eine mildere Art des Typhus. Trotz des lange andauernden Fiebers war meine Haushälterin immer bei hellem Bewusstsein; aber vielleicht sechs Wochen lang hatte sie nichts mehr gegessen; und die Fleischbrühe, die ihr der Arzt verordnete, nahm sie nur ungerne oder gar nicht. Jetzt habe ich volle Hoffnung, dass sie bei gehöriger Vorsicht nach einigen Wochen sich wi[e]der her stellen werde.

Indem ich Ihnen für Ihre Theilnahme danke, grüsse ich Sie herzlich und wünsche Ihnen volle Gesundheit.

Bern 15 Nov. 1876

Ihr ergebener Freund

Mittags

L. Schläfli.

Brunnadern 11c 
Theurer Freund!

Verzeihen Sie mir, dass ich die Antwort auf Ihren freundschaftlichen Brief so lange auf geschoben habe. Der Anfang der Vorlesungen, ich habe deren vier mit sechs Zuhörern, und widerholte Anfälle von Unwohlsein und daheriger Verstimmung machten, dass ich mir nicht getraute einen ordentlichen Brief zu schreiben. Nach langer Überlegung halte ich es dieses Mal für besser Ihre freundliche und grossmüthige Einladung als Gast zu Ihnen an den Comersee zu kommen nicht an zu nehmen. Wenn ich von Bern fort gehen will, so passt es am besten, wenn meine Haushälterin in der selben Zeit nach Basel zu Ihren Verwandten gehen kann; aber dieses Jahr ist sie schon zwei Male wegen Todesfall dort gewesen. Wir hatten schon lange im Sinne die Wohnung zu wechseln, haben es aber in diesem Jahre noch nicht zu Wege gebracht. Nächstes Jahr werden wir wohl eine andere Wohnung haben; und meine Haushälterin würde leichtere Gelegenheit haben, während meiner allfälligen Abwesenheit nach Basel zu gehen, wenn Sie dann auch wieder die Ferien am Comersee zubrächten und die Güte hätten mich als Gast zu sich zu nehmen.

Vor Empfang Ihres werthen Briefes hatte ich schon lange im Sinne Ihnen zu schreiben. Ich hätte nämlich schon im December dem Istituto Lombardo für die Ehre des Auftrages der Vertretung bei der Hallerfeier danken und über den Verlauf derselben berichten sollen. Ich dachte das in einem Briefe an Sie zu thun. Die Stadt Bern hatte ein ganz ungewöhnliches Gepränge veranstaltet. Schade war es aber, dass kein Mann von mit Haller verwandter Geistesrichtung, etwa ein Verehrer der Naturwissenschaft, sondern ein Rechtslehrer, die Festrede hielt und darin nur die Lebensgeschichte Hallers, ungefähr wie sie in der Festschrift zu lesen war, wieder gab. Nach meiner Ansicht bestund das Verdienst Hallers nicht nur in seiner staunenswerthen Gelehrsamkeit und Arbeitskraft, sondern mehr noch darin, dass er an der geistigen Bewegung des vorigen Jahrhunderts Theil nahm, in seinem kritischen wissenschaftlichen Sinne, den er, wie ein deutscher Redner beim Bankett sagte, nicht seiner Heimat, sondern dem Auslande verdankte. Aber die diametral-entgegengesetzte Richtung suchte Haller als einen der ihrigen dar zu stellen; die hiesige evangelische Gesellschaft veranstaltete auch dem Haller zu Ehren ein eigenes Fest und stellte ihn als Muster der Orthodoxie hin. Wer aber seine Gedichte gelesen hat, weiss dass Haller der Freiheit des Denkens nicht völlig fremd war. Thatsache ist nur, dass er in seinem Alter zur Vertreibung körperlicher Schmerzen viel Opium zu sich nahm und wahrscheinlich in Folge der dadurch bewirkten Geistesstörung an seiner zukünftigen Selig- 
keit verzweifelte. Beim Bankett ist es mir auf gefallen, dass den wenigsten Rednern die wissenschaftliche Bedeutung Hallers vor schwebte. Einer brachte gar einen Toast auf das Heimweh Hallers aus, das ihn von Göttingen nach Bern zurück geführt haben soll. Der erste, der in deutlicher Rede das wissenschaftliche Verdienst Hallers hervor hob, war der Geschichtsforscher Gonzenbach ${ }^{30}$; er sagte, er, Redner, sei zwar selbst nicht Naturforscher, und Haller sei nicht im eigentlichen Sinne Geschichtsforscher gewesen; aber einer von dessen Descendenten sei ein sehr tüchtiger und wahrheitsliebender Geschichtsforscher gewesen; und von diesem Descendenten aus könne man auf den wissenschaftlichen Sinn Hallers zurück schliessen. Moriz Schiff ${ }^{31}$ aus Genf (früher in Florenz) endlich wusste der Versammlung zu sagen, wer Haller gewesen sei, und dass er nichts für wahr aus gegeben habe, als was er selbst geprüft und richtig gefunden habe und dass er in dieser Eigenschaft noch die Gegenwart überrage.

In der Hoffnung dass Sie sich einer vortrefflichen Gesundheit erfreuen, grüsst Sie herzlich

Bern 15 Mai 1878

Ihr alter ergebener Freund L. Schläfli.

Nr. 5: Brief vom 24. Juli 1879, 3 Seiten

Theurer Freund!

Es ist ganz entsetzlich, dass ich Ihnen so lange nicht geschrieben habe. Sie haben mir nun schon widerholt Ihre werthvollen Arbeiten zu geschickt, und jedes Mal habe ich es verschoben Ihnen zu antworten. Ich habe mit einer Wohnungsänderung Missgeschick erfahren. Am 24 Jan. dieses Jahres schloss ich mit meinem jetzigen Hausherrn einen Miethvertrag ab für eine Wohnung, die mir sehr gefiel, mit Aussicht auf die Alpen, mit Gartenantheil und mit Wasserleitung in der Küche; leider auf ein ganzes $\mathrm{Jahr}^{32}$. Der Hausherr behauptet, damals noch nicht ans bauen gedacht zu haben. Mein Wohnungsrecht begann mit $\operatorname{dem} 1^{\text {sten }}$ Mai. Gegen das Ende des Aprils bei einer zufälligen Begegnung sagte mir der Hausherr, dass er in diesem Monat zu bauen an gefangen habe, und dass es ihm lieber wäre, wenn ich erst am $3^{\text {ten }}$ Mai bei ihm ein zöge. Weil ich aber in der alten Wohnung dem Nachfolger Platz machen musste, so zog ich am $1^{\text {sten }}$ Mai in der neuen Wohnung ein und hatte nur zwei Zimmer, Holzgemach und Keller zur Verfügung; zwei andere Zimmer und die Küche fehlten noch, und der zugewiesene Gartenantheil war mit Bauschutt bedeckt. Statt der 3 Tage wie ich zuerst glaubte, hiess es nun, in etwa 10 Tagen werde man mir die ganze Wohnung übergeben können. Es dauerte aber über 2 Monate, bevor ich von der Küche und 
vom Garten Gebrauch machen konnte. Ich hätte das alles geduldig ertragen, wenn ich nicht im Hause allerlei Feindseligkeiten erfahren hätte. Ich schlug dem Hausherrn vor, die Vertragszeit mit dem 1 Nov. zu beendigen. Das will er aber nicht, und ich bin genöthigt die Hülfe von Rechtsgelehrten an zu sprechen. Wie die Sache auch ab laufen mag, werde ich beträchtlichen Schaden haben.

Meine Haushälterin ist im Winter einige Male krank geworden; ich habe einen tüchtigeren Arzt herbei geschafft. Seit dem Frühling ist sie nicht mehr krank geworden. Ihretwegen kann ich aber die Wohnung nicht auf längere Zeit verlassen; denn wenn sie unwohl werden sollte, so wäre sie völlig verlassen und hätte niemand, den sie aus schicken könnte, um Hülfe zu suchen. Wenn Sie daher daran denken sollten, bei einem allfälligen Ferienaufenthalt am Fusse der Alpen mich in Ihre Gesellschaft auf zu nehmen, so könnte ich doch, so leid es mir thut, mein gegebenes Versprechen nicht erfüllen. Fern von Bern, wäre ich wegen meiner Haushälterin in beständiger Todesangst.

Ich bin zwar in diesem Jahre nie eigentlich krank gewesen; aber meine Gesundheit ist doch variabel. Das äussert sich besonders in der Arbeitsfähigkeit.

Ich hatte von Herrn Beltrami eine Antwort betreffend die Zeit, die für die Eingabe der zum Andenken an Herrn Chelini bestimmten Arbeiten gesetzt ist, erwartet $^{33}$. Können Sie mir vielleicht etwas darüber sagen?

Ich hoffe, dass Sie sich in bester Gesundheit befinden, und wünsche bald Nachricht von Ihnen und von allen Ihren Bekannten, die mir theuer sind, zu erfahren.

Es ist mir nicht wahrscheinlich, dass ich schon vor dem 1. Sept. Wohnung werde ändern können. Sollte es aber möglich sein, so wäre ich bereit, im Sept. zu Ihnen zu kommen; sonst aber im nächsten Jahre.

Es thut mir ordentlich leid, dass ich Ihnen solches schreiben muss; denn ich hange mit meinen Gedanken sehr stark an Ihnen. Seien Sie herzlich gegrüsst von Ihrem treuen Freund

Bern 24 Jul. 1879

L. Schläfli

Neufeldstrasse $200^{\circ}$

Nr. 6: Brief vom 25. August 1882, 4 Seiten

Verehrter und theurer Freund!

Ihr Brief vom $22^{\text {ten }}$ athmet so viele Freundschaft, dass ich fast nicht zu antworten weiss. Damit Sie sehen, dass meine Reise ganz gefahrlos war, will ich Ihnen dieselbe beschreiben. Von Madulein an führt ein guter Fussweg, der später ungefähr am Telegraphendraht erkennbar ist, in kurzer Zeit auf den Albula 
hinauf; und bergabwärts kann man an vielen Stellen die Windungen der Strasse ab kürzen. Ich hatte Sonnenschein genug um zu schwitzen. Den Rath des Herrn Pult ${ }^{34}$ befolgend, machte ich um 5 Uhr in Lenz Halt, wo ich im Postwirthshause übernachtete. Wegen des starken Andrangs von Fuhrwerken hatten die Wirthsleute viel zu thun, und ich musste meine Bitten oft widerholen, bis ich ein Zimmer bekam, wo ich Hemd und Stiefel wechseln konnte; und trotz eindringlichem Verlangen konnte ich kein Zimmer für mich allein bekommen; es hiess, es sei alles an gefüllt; und ich musste das Zimmer mit einem Kutscher theilen. Mit Tagesanbruch gieng ich von Lenz fort. Das Wetter war anfangs nicht übel; bald aber kamen hie und da kleine Regenschauer, die mich nicht belästigten, sondern nur den Schweiss ab hielten. Den Fussweg von Parpan nach Churwalden fand ich richtig, betrat auch anfangs den richtigen Fussweg von Churwalden nach Araschgen, kam aber bei der ersten Wegscheide zu hoch, verlor im Walde den Weg und gelangte wieder auf den rechten Weg, der mich an das zur Quelle Passugg gehörende vor kurzem neu erbaute Kurhaus führte (zwischen 10 und 11 Uhr), wo ich meinen frühern Studiengenossen, jetzigen Hausgenossen, Pfarrer Gobat, besuchte. Trotz seines hölzernen Beines begleitete er mich Nachmittags gegen 4 Uhr mit zwei andern Kurgästen (es waren etwa deren 30 da, und das Wasser soll dem von St. Moriz gleich sein) nach Chur hinunter. Um $5^{\text {h }} 20^{\text {' }}$ fuhr ich auf der Eisenbahn von Chur ab und langte um $10 \frac{1}{2}$ Uhr in Zürich an, wo ich in meinem gewohnten Gasthofe übernachtete. Um $6^{\mathrm{h}} 20^{\text {' }}$ Morgens nahm ich den Schnellzug nach Bern und langte hier um 10 1/2 Uhr Morgens an. Sie sehen hieraus, dass ich den vollen Genuss einer Reise durchs Gebirg hatte und doch nicht zu viele Zeit auf wandte. Im Postwagen wird man steif und muss sich langweilen; geht man in einer Berggegend zu Fuss, so kann man alles besser betrachten und kann sich mit den verschiedenartigen Pflanzen unterhalten.

Bei der Abreise hat mir die ruhige Überlegung gefehlt. Wenn Lenz das Ziel der Tagreise war, so war es früh genug, gegen $7 \mathrm{Uhr}$ ab zu reisen; wenn Sie dazu Lust hatten, so konnten Sie mich bis zum Hospiz begleiten, indem Sie zum Schutz gegen Zahnweh ein fazzoletto oder cache-nez um schlugen, und waren zur rechten Zeit wieder nach Zuz hinab gestiegen.

Meinen Freund Ris fand ich am Tage meiner Ankunft unverändert; er sieht aber immer besser aus, als er sich befindet. Obgleich er 1806 geboren ist, hat er noch schwarzen vollen Haarwuchs. Der Arzt hat ihm verordnet, Vormittag und Nachmittag eine bestimmte Anzahl von Stunden zu spazieren. Krank an Herz und Lunge, bedauert er es sehr, dass er nicht mehr arbeiten kann. Er war es, der, als ich Student der Theologie war und in Zeit von $1 \frac{1}{2}$ Jahren Vater und Mutter verloren hatte, ohne in irgend einer Beziehung zu mir zu stehen, mich auf suchte, 
mir Privatlehrthätigkeit in der Mathematik verschaffte, der mich über die Unmöglichkeit, die von den Eltern mir gegebene Bestimmung (Pfarrer zu werden) zu erfüllen, tröstete und mich belehrte, dass es meine Pflicht sei der Natur zu folgen und mich der Mathematik, zu widmen. Er hat überhaupt immer wie ein zweiter Vater für mich gesorgt.

Ich wünsche Ihnen auch, dass Sie für Ihre kostbare Gesundheit Sorge tragen. Dass Sie beinahe die Nacht mit dem Tage vertauschen, wird mit dem Klima zusammen hangen. Sie sind jetzt im kräftigsten Lebensalter und frei von Unpässlichkeit; Sie könnten sich daher vielleicht von Witterungseinflüssen unabhängiger machen; aber von der Art, wie das in Pavia geschehen sollte, habe ich keine Vorstellung. - Wie man sich in Bezug auf Gesundheitspflege irren kann, davon kann ich Ihnen gerade jetzt an mir ein Beispiel geben. Die Füsse brennen mich oft, besonders im Sommer; und das Übel hat mit den Jahren zugenommen. Unter dem Vorgeben (Vorwande), meine Fusssohlen taugen nicht dazu, schlug ich diesen Sommer ein Mal die Theilnahme an einer zweitägigen Bergfahrt aus. Dass das aber eine falsche Einbildung war, hat mir der Gang nach dem Engadin bewiesen; meine Füsse sind jetzt gar nicht beschädigt. Allerdings hatte ich kurze Strümpfe von ungebleichtem Hanf, der vielleicht entzündungswidrig ist, (sie waren ganz neu mir aus der Gegend von Basel gesandt worden), und gute schon durch Gebrauch erprobte Stiefel. Selbst die Strümpfe brachte ich fast unversehrt zurück, während sonst ein Tag hin reicht, um baumwollene Strümpfe zu brechen.

Ich achte Sie sehr hoch und habe Sie gerne. Mit herzlichem Grusse

Bern 25 Aug. Abends

Ihr ergebener

Brückfeld 237 c

\section{Nr.7: Brief vom 7.Januar 1888, 2 Seiten}

Theurer hochherziger Freund!

Ich sage Ihnen meinen herzlichen Dank für das werthe Schreiben, mit dem Sie mich an der Wende des Jahres erquickt haben.

Ein treuer Freund, dem ich sehr viel verdanke, Ris, Prof. der Philosophie, ist am 21 Dec. gestorben. Er war im Apr. 1806 geboren und übertrifft mich an Alter um 8 Jahre. Ich konnte genug sehen, wie seine Kräfte während einer Reihevon Jahren nach und nach ab nahmen. Er machte zuerst Aufenthalte an verschiedenen Orten im Berneroberlande; so viel ich mich aber besinne, kam er immer unbefriedigt zurück. Der härteste Schlag für ihn war, als sein Arzt durchaus ver- 
langte, dass er wegen seines Brustleidens auf höre, Vorlesungen zu halten. Dann kam die Abnahme der Schärfe der Augen hinzu, so dass er nicht mehr lesen konnte und sich unglü[c]klich fühlte.

Ich habe nun den Verlauf des Alters deutlich vor Augen und kann gar nicht erwarten, dass es mir besser gehn werde als meinem Freunde, der physisch stärker war und z. B. nie an Magenübeln litt. Ich habe alle Ursache, Ihnen für Ihre freundschaftlichen Räthe, die ich zum Theil schon befolge, zu danken, und will hoffen, dass ich mein Leben bis zum nächsten Herbste fort setzen könne. Aber der Rath, dass ich mich durch gesprächigen Umgang mit andern erheitere, ist für mich kaum möglich aus zu führen. Ein Mal habe ich keine gesellschaftliche Anlage, und zum andern sind meine meisten Bekannten schon todt.

Es war überflüssig, dass ich eine Vermuthung ... ${ }^{25}$

\section{Nr. 8: Brief vom 9.Juli 1888, 3 Seiten}

Theurer Freund!

Ich antworte wohl spät auf Ihr werthes Schreiben vom 23. Jun. Mein rheumatisches Übel hat seit meiner Wohnungsänderung vom 1. Mai, besonders im kalten und regnerischen Juni merklich zu genommen, und Bekannte hatten mir es als unabweisliche Forderung hin gestellt, dass ich die Thermen von Baden im Aargau gebrauche, was mir wegen der Langeweile, die ich aus stehn müsste, schrecklich vor kam. Ich wollte zuerst den Arzt um Rath fragen, dem ich natürlich kein Wort von den Thermen sagte. Ich war glücklich, als er mir nur warme Sodabäder verordnete. Ich habe deren erst zwei genommen, ohne irgend eine Wirkung zu spüren, werde aber fleissig fort fahren. Ich bin zu steif in den Bewegungen, um eine Reise zu wagen, obschon ich sehr gerne nach Piora gekommen wäre.

Ich sage Ihnen meinen herzlichen Dank für die zugesandte Abhandlung, deren Anfang ich schon in den Annali gelesen hatte; und es freut mich, dass Sie der willkürlichen Beschränkung der Functionen durch den Begriff der coupures (Hermite und Goursat) Ihre Zustimmung versagen ${ }^{36}$.

Sie werden Oswald Heer's Buch, die Urwelt der Schweiz, hoffentlich wohl erhalten, schon empfangen haben; es ist für Herrn und Frau Franconi bestimmt. Weil die Brieflöcher am Sonntag nicht geleert werden, so kommt dise Anzeige, der Sendung, die wegen der an der Zollstätte nothwendigen Öffnung durch den Buchhändler geschah, nach gehinkt. Entschuldigen Sie die Wahl des Buches, die Ihnen sonderbar vor kommen mag, durch meinen beschränkten Gedankenkreis; ich habe dasselbe nur bei Frau Landammann Sidler sel. gesehen, die sich lobend darüber aus sprach. Ich hatte zwei Male Gelegenheit den Verfasser kennen 
zu lernen. Das erste Mal sprach er über geologische Perioden; er nahm deren 6 an, indem er sich zu meiner Verwunderung durch Gen. 1 bestimmen liess. Nach ihm sprach der Geolog Escher ${ }^{37}$ über den selben Gegenstand, ohne auf irgend einen alten Versuch das Dasein der Welt zu erklären Rücksicht zu nehmen, nach meiner Überzeugung auf die einzig einem Naturforscher gezimende Weise. Beim zweiten Male hatte Heer schon eine schwache Brust; er sprach über die Coniferengattung Sequoja, die früher, den fossilen Überresten nach zu urtheilen, eine weite Verbreitung hatte und durch vi[e]le Arten vertreten war, aber jetzt, in einer einzigen Art, nur noch in Californien existiert; er wis damals auch Zweige des noch lebenden Baumes vor. Kurz, ich bekam von der unermüdlichen Thätigkeit und der Wahrheitsliebe des Mannes eine hohe Vorstellung; und dises Buch wird auch ein beredtes Zeugniss dafür ab legen.

Ich empfehle mich dem Wohlwollen Ihrer Angehörigen, wünsche Ihnen Erholung in Piora und grüsse Sie herzlich,

Bern 9 Jul. 1888

Ihr alter Freund

Bolligenstrasse 18.

L. Schläfli

Nr. 9: Brief vom 3. August 1889, 2 Seiten

Verehrter Freund!

Gesicht und Gehör sind zu schwach, als dass ich allein eine Fahrt im Dampfwagen wagen möchte. Voriges Jahr als ich in Thun einen Krankenbesuch machte, hätte ich, zurück gekommen, bei Bern den Weg von der Station zu meiner Wohnung ohne die Hülfe meiner Haushälterin, die mich nach Thun begleitet hatte, nicht gefunden; und in den drei Wochen meines Aufenthalts in Baden (Merz-Apr.) war sie immer bei mir; nur am Ende der Cur machte ich eine Fahrt (Dauer kaum 1 Stunde) allein nach Zürich, sorgte aber dafür; dass ich Tags in Baden an kam, um den Weg in den Gasthof zu finden. Ich habe disen Sommer Vorlesungen gehalten und konnte zwei Stunden nach einander ohne Beschwerde sprechen. Seit ich in Baden gewesen bin, kann ich die Arme frei gebrauchen; nur das gehn ist, obgleich ich gut schreiten kann, noch so, als ob ich schon weit gegangen wäre; im übrigen bin ich wohl. Sie werden bei diser Sachlage begreifen, dass ich Ihre freundliche Einladung nach Baveno zu kommen mich nicht entschliessen kann an zu nehmen.

Die Rendiconti dell'Istituto Lombardo sind hier nicht zu haben, und die andere Arbeit des Herrn Ascoli in XVIII Mem. lincee, die ich von der Bibliothek der schw. naturf. Ges. geholt habe, bewegt sich so sehr in Cantors Anschauungen, 
dass ich es nicht wagte sie zu lesen ${ }^{38}$. Schon als im vorigen Jahre Mittag Leffler mir dessen Sätze über Punktmengen mit theilte, konnte ich nur den Anfang verstehn, und dann ward ich schwindlig.

Dass mir in Ihrem werthen Briefe vom 31. Mai etwas dunkel gebliben sei, glaube ich nicht. Das Wort Zweck habe ich in unbefangenem Sinne gebraucht. Fast alles, was ich über den Gegenstand sagen könnte, sagen Sie selbst; ich könnte Sie höchstens noch auf den Unterschid zwischen einer rein geometrischen Anschauung und einer geometrischen, die mit analytischen Elementen gemischt ist, aufmerksam machen. Da Sie mir weit überlegen sind, so thut es mir Leid, dass Sie in Bezug auf mein Urtheil einen so starken Ausdruck gebraucht haben. Die Herren Maggi ${ }^{39}$, Bertini, Beltrami sind Ihnen näher und competentere Richter als ich bin.

Ich lasse Herrn Maggi und Frau grüssen.

Mit herzlichem Gruss und Handschlag

Bern 3 Aug. 1889

Ihr ergebener L. Schläfli.

Ausgewählte Akten zu Ludwig Schläfli in Berner Archiven

Nr. 1: Schreiben des Erziehungsdepartementes des Kantons Bern an den Verwaltungsrat des Progymnasiums Thun vom 5.Juni 1843

Quelle: Missiven-Protokoll des Erziehungs-Departementes, Staatsarchiv des Kantons Bern BB III b 1075, S. 199-200

Nachdem wir sowohl von dem mit Ihrem Schreiben d. d. 8 Mai lezthin an uns gelangten Berichte über den Fortgang Ihrer Anstalt im Schuljahre 1842-43 Kenntniß genommen, als das Urtheil unserer Abgeordneten über das Ergebniß der Schlußprüfung vom 24-26 April lezthin angehört haben, finden wir uns im Allgemeinen durch die Leistungen der Lehrer, wie der Schüler befriedigt und stimmen ganz der lobenden Anerkennung bei, die Sie in dieser Beziehung bereits am öffentlichen Schulfeste ausgesprochen haben.

Die besondern Bemerkungen, die wir Ihnen zu Handen der betreffenden Lehrer mitzutheilen haben, beschränken sich auf folgende.

Erfreulich ist die Wahrnehmung gewesen, daß Herr Hunziker ${ }^{40}$ die Rügen, zu denen sein vorjähriges, wie überhaupt seine frühern Prüfungen Anlaß gegeben, zu Herzen genommen und seine Unterrichtsmethode auf eine augenscheinlich vortheilhafte Weise geändert hat. Indem wir ihm die hierseitige Anerkennung seiner Bemühungen aussprechen, wünschen wir nur, daß er Geographie und Geschichte mehr in ihrer wechselseitigen Beziehung aufeinander vortragen, im erstern Fache bei sich darbietender Gelegenheit auf historische Ereignisse hinweisen, im leztern bei seinen Erzählungen, wo es sich thun läßt, die Landcarte zur Hand nehmen möchte.

Dagegen bedauern wir, unsere vorjährige Bemerkungen über die Mängel des Unterrichtes in der Mathematik und Naturlehre wiederholen zu müssen. Herr Schläfli hielt seinen Unterricht fortwährend zu hoch und ertheilt ihn unpractisch, docirt zu viel, frägt und wiederholt zu wenig, so daß nur die begabtesten Schüler ihm folgen können und der 
grös[s]te Theil derselben wenig Befriedigendes leistet. Auch hat es ihm an der Prüfung namentlich in der Mathematik durchaus an der für dieses Fach ganz besonders erforderlichen Ruhe, Unbefangenheit und Sicherheit gefehlt. Nachdem Herr Schläfli nun schon mehrere Male auf die Fehler seiner Unterrichtsweise ohne sichtbaren Erfolg aufmerksam gemacht worden ist, müssen wir ihn noch einmal alles Ernstes auffordern, unsern Bemerkungen in Zukunft Rechnung zu tragen, indem wir auf die Länge nicht zugeben könnten, daß in Unterrichtsfächern von der Wichtigkeit, wie Mathematik und Naturlehre, bei den gründlichen Kenntnissen des Lehrers infolge der practischen Methode desselben von den Schülern so wenig geleistet wird.

Indem wir über die Leistungen im Französischen, ...

Nr. 2: Schreiben des Verwaltungsrats des Progymnasiums Thun an den damaligen Direktor Gottlieb Stähli des Progymnasiums vom 21. August 1846

Quelle: Protocoll der Sitzungen des Verwaltungsraths des Progymnasiums in Thun, Stadtarchiv Thun AN $16.12 / 5$, S. $106 \mathrm{f}$.

Der Verwalt. Rath des Progymnasiums hat zu seinem großen Leidwesen vernehmen müßen, daß die Unordnung und Zuchtlosigkeit der Schüler in den Unterr. stunden des H. Schläfli einen so hohen Grad erreicht haben, daß der Unterr. fast zur Unmöglichkeit geworden ist; und demnach die Schüler nicht nur in den betreffenden wißenschaftl. Fächern keine Fortschritte machen können, sond. auch sittlich zu verwildern Gefahr laufen. Da nun die Aufsichtsbehörde nicht durch sorgloses Gehenlaßen den gedeihlichen Fortgang der Anstalt gefährden darf, so haben wir vor diesem in Erfahrung gebrachten höchst folgereichen Übelstande nicht die Augen schließen können, sondern nach aufhabender Pflicht über Mittel u. Wege uns berathen, wie wir ihn beseitigen helfen und dem Geiste der Ordnung, des Gehorsams, der Ehrerbietung und des wißenschaftlichen Eifers wieder Eingang verschaffen könnten. Einmüthig haben wir dabei die Nothwendigkeit erkannt, um so mehr Ihre Mithülfe zur Erreichung unsers Zweckes in Anspruch zu nehmen, als wir bei der Berathung, der Sie nicht beiwohnten, Ihres Rathes entbehrten. Ja wir wünschen, daß zunächst nur Sie sich bei der Sache direct betheiligen und dieselbe ins heilsame Geleise zurückzubringen versuchen möchten. Zu dem Ende ersuchen wir Sie - bei aller Anerkennung deßen, was Sie schon gethan haben mögen - noch ununterbrochener als bisher dem betreffenden Lehrer in Handhabung der Disciplin mit Rath und That kräftig beizustehen, und namentlich eine längere Zeit so oft es sich nur thun läßt, jedoch ohne Aussetzung der eigenen Unterrichtsstunden, denjenigen von H.Schläfli beizuwohnen.

Nicht nur hoffen wir, daß Sie dieser unserer Aufforderung zu Erfüllung einer, wie wir uns nicht verhehlen, sehr schweren Directoral-Amtspflicht bereitwillige Folge geben werden, sondern haben auch das Vertrauen, daß es Ihrem pädagogischen Geschick und Ihrem Eifer gelingen werde, Ordnung und Zucht auch in den Stunden dieses in Handhabung der Disciplin von jeher schwachen Lehrers wiederum zur Herrschaft zu bringen, und dadurch die Schüler der Gefahr großen sittlichen und wißenschaftlichen Schadens zu entziehen und den treuen, wißenschaftlich so tüchtigen Lehrer von unerträglicher Qual zu befreien.

Erfüllt von dieser Hoffnung u. diesem Vertrauen legen wir vorläufig die Aufgabe zu deren gesegneter Lösung wir Ihnen den Beistand Gottes wünschen, in Ihre Hände u. verharren hochschätzungsvoll

Die Unterschriften. 
Nr. 3: Schreiben der Erziehungsdirektion des Kantons Bern an Herrn Ludwig Schläfli, Lehrer am Progymnasium in Thun, vom 1. April 1847

Quelle: Missiven-Protokoll der Erziehungs-Direktion, Staatsarchiv des Kantons Bern BB III b 1088, S. 283

Am 31 März letzthin hat der Regierungsrath beschlossen, die vacanten Lehrstühle der Mathematik und Physik einstweilen nicht definitiv durch Professoren zu besetzen, sondern diese Fächer durch Docenten vortragen zu lassen.

Es hat daher Ihre Bewerbung ${ }^{10}$ um den Lehrstuhl der Mathematik nur in so weit Berüksichtigung finden können, daß Sie hiermit eingeladen werden, als Docent in diesem Fache an der Hochschule aufzutreten, wobei Ihnen nach $\S 38$ des Hochschulgesetzes das Maximum des Docentenhonorars, L 400 jährlich, in Aussicht gestellt wird.

Obschon dieses Anerbieten allerdings nicht ganz geeignet ist, Sie zu bewegen, Ihre fixe Stelle in Thun aufzugeben, so setze ich doch grossen Werth darauf, daß die Hochschule Sie als Lehrer gewinne, und werde mein Möglichstes thun, um Ihre oeconomische Lage auf andere Weise zu verbessern, bis der wohl nicht sehr ferne Zeitpunct kommen wird, wo Sie eine festere, Ihren Kenntnissen angemessenere Stellung an der Hochschule erhalten werden.

Indem ich also wünsche, daß Sie der an Sie ergehenden Einladung entsprechen möchten, richte ich das Ansuchen an Sie, mir Ihren daherigen Entschluß mit möglichster Beförderung mitzutheilen und, falls er bejahend ausfällt, mir auch die Ankündigung der Vorlesungen zuzusenden, die Sie zu halten gedenken, zugleich aber auch sofort Ihre Stelle in Thun aufzukünden.

Nr. 4: Vortrag der Erziehungsdirektion des Kantons Bern an den Regierungsrat anläßlich der Pensionierung von Ludwig Schläfli vom 28.Oktober 1891

Quelle: Staatsarchiv des Kantons Bern BB III b 26011

Hr. Dr. Ludwig Schläfli v. Burgdorf, ordentl. Profeßor der Mathematik an unserer Hochschule, sieht sich infolge Abnahme der geistigen und leiblichen Kräfte genöthigt, in den Ruhestand zu treten und sucht um Gewährung einer Pension nach. - Hr. Schläfli wurde geboren am 15. Jan. 1814, und es ist wohl eine Seltenheit, daß ein Gelehrter mit beinahe ungeschwächter Kraft bis zu einem so hohen Alter gewirkt hat. Derselbe hat Theologie studirt, wirkte dann als Lehrer am Progymnasium Thun, seit 1847 als Privatdocent, seit 1853 als außerordentl. und seit 1872 als ordentl. Profeßor der Mathematik an der Hochschule. Trotzdem er sich als Gelehrter eines großen, weitverbreiteten Ruhmes erfreute und unserer Universität zur Zierde gereichte, war seine Besoldung lange Jahre hindurch eine äußerst kärgliche, zuerst fr. 580., dann lange Zeit fr. 1200.-, dann fr. 1400. bis 1872, 1873 fr. 2000.-, v. 1873 an fr. 3000 . und seit 1880 fr. 4000, das Minimum eines ordentl. Profeßors. Nur bei seiner bekannten Einfachheit und Bescheidenheit war es Hrn. Schläfli möglich mit einer solchen Besoldung auszukommen. Es erscheint daher billig demselben nun auch den üblichen Maximal-Ruhegehalt v. fr. 3000. auszurichten. 
Wir beehren uns, Ihnen zu beantragen, Hrn. Prof. Dr. L. Schläfli eine Pension v. fr. 3000.per Jahr zu bewilligen und zwar v. 1. Jan. 1892 an.

Bern, 28. Oct. 1891.

Der Erziehungsdirector:

Dr.Gobat

\section{Anmerkungen}

Wir danken Herrn Prof. Alberto Gabba (Betreuer des Casorati-Nachlasses), Herrn Hans Schmocker (Adjunkt am Staatsarchiv Bern) und Herrn Otto Zimmermann (Archivar am Burger- und Stadtarchiv Thun) für ihre stete Hilfsbereitschaft bei der Abfassung dieser Arbeit.

1 Angaben zu Schläflis Vorfahren sowie zu weiteren bedeutenden Persönlichkeiten der Schläfli-Familie findet man bei Keiser 1962, S. 104f., 114f. sowie bei Leibundgut 1978, S. 9 f. Leibundgut erwähnt in seiner Anmerkung 3 einen «Stammbaum Schläfli», der sich im Burgerarchiv Burgdorf befinden soll.

${ }^{2}$ Vgl. hierzu Brief Nr. 6, Abschnitt 3. Schläflis Vater starb 1832, seine Mutter 1833; die bei Graf 1895, S. 124, wiedergegebene Mitteilung des Herrn Dr. Ris kann somit in der bei Graf wiedergegebenen Form nicht zutreffen.

${ }^{3}$ Im Jahre 1852 wurde der alte Franken durch Multiplikation mit 1,44 in den neuen schweizerischen Einheitsfranken umgerechnet. Vgl. Feller 1935, S. 179.

${ }^{4}$ Burgerarchiv Thun AN 125 1/3, Schulraths-Manual Nr. IV, S. 165-172, speziell S. 172.

5 Vgl. Graf 1895, S. 123.

${ }^{6}$ Schläfli wurde am 5.9.1838 ins Predigtamt aufgenommen (Staatsarchiv Bern, Manual des Reg. Rates 62, S. 454f.).

7 Protocoll der Sitzungen des Verwaltungsraths des Progymnasiums in Thun $=$ SAT AN 16.1 2/5, S.24. (Die Signatur SAT AN bedeutet: Stadtarchiv Thun, Akten-Nummer.)

${ }^{8}$ SAT AN 16.1 2/5, S. 4 ff., S. 7. Nach den Akten der Bernischen Erziehungsdirektion (StAB BB III b 1056, S. 469 f.) meldeten sich neben Schläfli noch 6 weitere Bewerber anläßlich der Stellenausschreibung.

9 Vgl. Trepp 1938, S. 69-78, S. 165 f. (speziell: S. 73 ff.), sowie SAT AN 16.1 2/5, S. 15-17, S.19f., S. 21, S. 33 usw. Aus den Protokollen des Verwaltungsrates ergibt sich auch, daß Schläfli an der Schule eine Mineraliensammlung einrichtete (vgl. ebenda S. 28f., S. 68 und S. $85 \mathrm{f}$.$) .$

10 Vgl. hierzu Weilenmann 1958, S. $192 \mathrm{f}$.

11 SAT AN $16.12 / 5$, S. 77.

12 Graf 1895, S. $125 \mathrm{ff}$.

13 Die erste Zahl findet man bei Burckhardt 1948, S. 20, und Burckhardt 1975, S. 171; die zweite bei Feller 1935, S. 274.

14 Aus den Akten im Berner Staatsarchiv ergibt sich, daß Schläfli seine Thuner Stelle Ende 1847 verließ (StAB BB III b 1088, S. 342f.) und für das Sommersemester 1847 bereits Vorlesungen ankündigte (ebenda S. 310).

15 Vgl. sein Schreiben an die Erziehungsdirektion aus dem Jahre 1853 bei Graf 1895, S. $129 \mathrm{f}$.

16 Vgl. Feller 1935, S. 223.

17 Friedrich Ris (1806-1887), Lehrer der Stadtschule zu Burgdorf 1837, Studium in Heidel- 
berg, Berlin 1842-1844, Tübingen, Professor der Philosophie an der Berner Hochschule 1846-1881.

18 Vgl. Graf 1895, S. 130 f.; 1898, S. 244.

19 Für die genauen Angaben zu dieser und den weiter unten erwähnten Schriften vergleiche die Bibliographie.

${ }^{20}$ Nach Graf 1895, S. 135, aß Schläfli während eines ganzen Jahres bei der Familie Graf jeweils zu Mittag.

21 Für nähere Angaben zu Casorati vgl. Neuenschwander 1978.

22 Vgl. hierzu Graf 1916, Brief Nr. 28.

${ }^{23}$ Den ersten Teil der Reise schildert Schläfli in einem vorangegangenen Brief vom 12.10. 1872 aus Airolo. Das in diesem Brief vermerkte Datum «12. Sept. $1872 »$ ist falsch. Aus Briefentwürfen von Casorati an Beltrami (vom 31.8.1872) und an Schwarz (vom 1.9.1872 und 6.10.1872) ergibt sich nämlich, daß Schläfli von Ende August bis anfangs Oktober bei Casorati weilte.

${ }^{24}$ Oswald Herr (1809-1883), Naturforscher, besonders Paläobotaniker, auch Entomologe, ab 1835 Direktor des botanischen Gartens und Ordinarius für Botanik in Zürich. Sein Werk «Die Urwelt der Schweiz» erschien in 1. Auflage im Jahre 1865 und in 2. Auflage im Jahre 1879 in Zürich.

25 Weitere Angaben zum Gesundheitszustand der Haushälterin, der sich seit 1880 wesentlich verbessert hat, findet man in Schläflis Briefen vom 13.10.1876, 15.11.1876, 6.1.1877, 14.8.1877, 7.8.1880 und 2.8.1881.

26 Vgl. Feller 1935, S. 168 f., S. 273 f., S. 319 und S. 369.

27 Vgl. Brief Nr. 2 und 4 sowie die unpublizierten Briefe vom 4.11.1879, 7.8.1880, 2.8.1881, 17.9.1881, 8.8.1882 usw.

${ }^{28}$ Ob der Rheumatismus Schläflis im Februar 1886 oder 1887 einsetzte, ist aus dem Briefwechsel nicht klar ersichtlich, da Schläfli in einem späteren Brief an Casorati vom 25. April 1888 schreibt: «Der Rheumatismus, der mich am Ende febr. 87 plötzlich befiel und im Laufe des Sommers sich ein wenig verminderte, hat in diesem lange dauernden Winter nach und nach zu genommen, ...».

${ }^{29}$ Verwandte und Bekannte von Casorati, die jeweils mit ihm an den oberitalienischen Seen weilten.

30 August Gonzenbach (1808-1887), Justizsekretär und Staatsanwalt in St. Gallen, eidgenössischer Staatsschreiber 1833, war nach seinem Rücktritt 1847 Berner Bürger, Großrat und Nationalrat geworden und widmete sich umfassenden historischen Studien.

31 Moritz Schiff (1823-1896), enger Freund von Schläfli, Professor der vergleichenden Anatomie in Bern 1855-1862, dann der Physiologie an der Universität Florenz 18631876 und in Genf von 1876 bis zu seinem Tode.

32 Die Wohnungsmiete pro Jahr betrug Fr. 620. Vgl. hierzu den unpublizierten Brief von Schläfli vom 16.8.1879 mit weiteren Angaben zum Wohnungswechsel.

${ }^{33}$ Die "Collectanea mathematica in memoriam D. Chelini» erschienen im Jahre 1881 in Mailand. Schläflis Beitrag trug den Titel «Einige Bemerkungen über die Laméschen Funktionen ». Vgl. Schläfli 1950-1956, Bd. 3, S. 300-308.

${ }^{34}$ Die Familie Pult betrieb in Zuoz eine Pension, in der sich Casorati und andere Mathematiker oft in den Ferien aufhielten. Vgl. hierzu Graf 1916, Briefe Nr. 18-21, Nr. 38, sowie die unpublizierten Briefe von Schläfli vom 9.6.1877 und 3.7.1877. 
${ }^{35}$ Für die Fortsetzung des Briefes vgl. Neuenschwander 1978, Brief Nr. 32.

36 Vgl. F. Casorati, Sopra le coupures del sig. Hermite, i Querschnitte e le superficie di Riemann, ed i concetti d'integrazione sì reale che complessa (Annali di Matematica, Serie II, Vol. 15, 1887; Serie II, Vol. 16, 1888) = Opere, Bd. 1, S. 385-418.

37 Arnold Escher von der Linth (1807-1872), Professor für Geologie an der Hochschule Zürich 1852 und am Eidgenössischen Polytechnikum 1856. Verfasser mehrerer Werke zur Geologie der Schweizer Alpen.

${ }^{38}$ Für nähere Angaben zu den beiden Arbeiten vgl. Graf 1916, Brief Nr. 49.

39 Gian Antonio Maggi (1856-1937), Professor für theoretische Mechanik an den Universitäten Pisa und Mailand, Schwiegersohn von Casorati (Hochzeit mit Eugenia Casorati am 23.9.1883).

40 Für nähere Angaben zu den Lehrern am Progymnasium vgl. Trepp 1938.

\section{Bibliographie}

Burckhardt, Johann Jakob, Der mathematische Nachlass von Ludwig Schläfli (1814-1895) an der Schweizerischen Landesbibliothek. In: Mitteilungen der Naturforschenden Gesellschaft Bern aus dem Jahre 1942, S. 1-22.

Burckhardt, Johann Jakob, Ludwig Schläfli. Beihefte zur Zeitschrift «Elemente der Mathematik». Nr. 4, Basel 1948.

Burckhardt, Johann Jakob, Schläfli, Ludwig. In: Dictionary of Scientific Biography, Bd.12, New York 1975, S. 170-173.

Feller, Richard, Die Universität Bern 1834-1934, Bern/Leipzig 1935.

Graf, Johann Heinrich, Ludwig Schläfli. 1814 bis 1895. In: Mittheilungen der Naturforschenden Gesellschaft in Bern aus dem Jahre 1895, S. 120-203.

Graf, Johann Heinrich, Der Briefwechsel zwischen Jakob Steiner und Ludwig Schläfli. In: Mittheilungen der Naturforschenden Gesellschaft in Bern aus dem Jahre 1896, S. 61-264.

Graf, Johann Heinrich, Die Exhumirung Jakob Steiner's und die Einweihung des Grabdenkmals Ludwig Schläfli's. In: Mittheilungen der Naturforschenden Gesellschaft in Bern aus dem Jahre 1897, S. 8-24.

Graf, Johann Heinrich, Ludwig Schläfli. 1814-1895. In: Sammlung Bernischer Biographien, Bd. 3, Bern 1898, S. 241-248.

Graf, Johann Heinrich, Briefwechsel von Ludwig Schläfli mit Arthur Cayley. In: Mitteilungen der Naturforschenden Gesellschaft in Bern aus dem Jahre 1905, S. 70-107.

Graf, Johann Heinrich, Briefwechsel Ludwig Schlaeflis mit C. W. Borchardt. 1856-1877. In: Mitteilungen der Naturforschenden Gesellschaft in Bern aus dem Jahre 1915, S. 50-69.

Graf, Johann Heinrich, La corréspondance entre Ludwig Schläfli et des Mathématiciens Italiens de son époque. In: Bollettino di bibliografia e storia delle scienze matematiche 17 (1915), S. 36-40, S. 81-86, S. 113-122; 18 (1916), S. 21-35, S. 49-64, S. 81-83, S. 113$121 ; 19$ (1917), S. 9-14, S. 43-49, S. 65-73.

Häusermann, Albert, Über die Berechnung singulärer Moduln bei Ludwig Schläfli, Bearbeitung der Manuskripte und Darstellung der Hauptresultate, Dissertation: Zürich 1943.

Keiser, Walter, Erinnerungen an den Kunstmaler Eugen Schläfli. In: Burgdorfer Jahrbuch 1962, S. 104-115. 
Leibundgut, Fritz Albrecht, Die Reisen des Dr. med. Alexander Schläfli von Burgdorf. In: Burgdorfer Jahrbuch 1978, S. 9-99.

Linder, Arthur, Ludwig Schlaefli über den physikalischen Raum. Ein Beitrag zur Vorgeschichte der Relativitätstheorie. In: Commentarii Mathematici Helvetici 3 (1931), S. $148-150$.

Loria, Gino, Commemorazione del compianto Socio prof. Placido Tardy. In: Atti della R. Accademia dei Lincei. Rendiconti, Cl. fis., mat. e nat. 24 (1915), S. 505-531 [mit zwei Briefen von Schläfli an Tardy auf S. 519-531].

Neuenschwander, Erwin, Der Nachlaß von Casorati (1835-1890) in Pavia. In: Archive for History of Exact Sciences 19 (1978), S. 1-89.

Rytz, Walter, Professor Ludwig Schläfli als Botaniker. In: Mitteilungen der Naturforschenden Gesellschaft in Bern aus dem Jahre 1919, S. 213-220.

Schläfli, Ludwig, Gesammelte Mathematische Abhandlungen. Herausgegeben vom SteinerSchläfli-Komitee der Schweizerischen Naturforschenden Gesellschaft, 3 Bde., Basel 1950-1956.

Schlaginhaufen, Otto, Der Schädel des Mathematikers Ludwig Schläfli. In: Mitteilungen der Naturforschenden Gesellschaft Bern aus dem Jahre 1931, S. 35-66.

Trepp, Martin, 100 Jahre Progymnasium Thun. 1838-1938. Festschrift zur Jahrhundertfeier. Thun 1938.

Weilenmann, Heinz, Berner Professorenwahlen in vergangenen Tagen. In: Berner Zeitschrift für Geschichte und Heimatkunde 1958, S. 192-196.

\section{Summary}

Ludwig Schläfli is one of the few important Swiss mathematicians from past centuries who troughout his life taught only in Switzerland. ln my paper are puplished nine of his letters of cultural and biographical value from the Casorati-Nachlass, supplementing my previous mathematical study on this Nachlass. I also take the opportunity to clarify some little known periods of Schläfli's life, correct some mistakes in former biographies, and publish four documents out of Bernese archives.

PD Dr. Erwin Neuenschwander

Mathematisches Institut

Freiestraße 36

8032 Zürich 